continue in the same orbit as the comet and give rise to meteor showers when they penetrate the Earth's atmosphere.

Some meteor showers have never been associated with comets - the strong December shower known as the Geminids, for example. Here the best that can be done is to assume that the parent comet has completely disintegrated, or that either the comet or the meteor stream has been perturbed out of its original path by close approaches to the major planets.

Most meteors, it seems, do not belong to any of the major showers, and make up what is known as the sporadic meteor flux. These meteors are now widely believed to represent the end product of the perturbation and dispersion of meteor streams.

The rocky bodies from space which succeed in penetrating the atmosphere and reach the surface without being completely burnt up are termed meteorites, and seem to have quite a different origin to the small particles making up meteor streams and the sporadic meteor flux, which burn up completely in the upper atmosphere as shooting stars. The evidence for this is that no meteorite has ever been seen to fall from a recognized meteor shower and the fact that their orbits, calculated from the reports of eye witnesses, are similar to the orbits of asteroids. Another factor in favour of an asteroidal origin for these bodies is in their structure, which suggests that they are fragments of a large body of asteroidal size.

The extent to which smaller meteoric particles of the asteroidal type exist, possibly making up an appreciable part of the sporadic meteor flux, is still an open question. In recent years several attempts have been made to recover meteoric particles from space using collecting devices on rockets and satellites, but so far it has proved impossible to separate the extra-terrestrial component from terrestrial contamination.

\section{Non-porous Ceramics}

\section{from our Materials Science Correspondent}

Ceramrcs made in the usual way by sintering powders generally retain 5 per cent or more of residual porosity. In 1962, R. L. Coble at the General Electric Research Laboratories in Schenectady recognized the essential role of grain boundaries in densification during the sintering process, and from this insight developed a technique of eliminating pores entirely. Pores contract and vanish by dispatching vacancies through the ceramic, but vacancies can move at useful speeds only along grain boundaries. The trick therefore is to maintain a small grain size and thus keep a large population of boundaries; it is essential to prevent pores from being marooned in the interior of large grains. While investigating the sintering of alumina, Coble and his colleagues found that by adding small quantities of magnesia, which collects at grain boundaries, to the alumina, they were able to inhibit the motion of boundaries and thus enhance densification. The product is free of pores and therefore translucent (hence its trade name, 'Lucalox'). Later developments in the United States and Britain led to pore-free polycrystalline yttria and magnesia; the latter is as transparent as clear glass. These pore-free oxides have a number of industrial applications which depend chiefly on their optical properties.
A similar technique has now been applied at the Central Electricity Board Research Laboratory to barium titanate, industrially important for its ferroelectric behaviour and for certain associated anomalies in its electrical conductivity (C. A. Miller, Journal of Materials Science, 3, 436; 1968). The problem here is that the electrical characteristics of barium titanate are highly sensitive to small concentrations of impurity, especially if these segregate at grain boundaries, for then the contaminated boundaries are apt to form a network of lower resistivity; for some applications this is desirable, but not for the one with which Miller was concerned. Accordingly, he sought an additive or dopant which can be removed after the sintering process. He added a few parts per thousand of sugar to the barium titanate powder before cold-pressing. During sintering in vacuo, the sugar charred to produce a fine distribution of carbon particles which effectively inhibited grain growth, so that the mean grain size remained less than $1 \mu \mathrm{m}$. The porosity was normally reduced to about 1 per cent by volume after 200 hours' sintering at $1,350^{\circ} \mathrm{C}$. After sintering, the compacts were briefly heated in air at $100^{\circ} \mathrm{C}$ to remove the carbon by oxidation. (It would be interesting to know the precise mechanism involved here: carbon is insoluble in barium titanate-which explains its use in this system-and it is not obvious how either carbon or carbon dioxide can diffuse through the dense compact.)

The almost pore-free fine-grained compacts have an exceptionally high permittivity (C. A. Miller, Brit. J. Appl. Phys., 18, 1689; 1967). The material can be used to make high tension insulators which are particularly resistant to electrical breakdown, because the small but reproducible and stable leakage current through a string of insulators assures a smooth potential gradient along the string. In a tailpiece to his recent paper, Miller mentions some attempts to apply the same principle to the production of electrically conducting ceramics, in which the additive is left in position after sintering. He reports success with silver-doped alumina.

\section{Radiation Carcinogenesis}

\section{from our Radiobiology Correspondent}

ONE way of interpreting the carcinogenic effect of radiation is to regard it as a special form of sub-lethal damage suffered by irradiated cells. In general, sublethal damage is wholly or partly repaired during the interval before the next division, a few hours in the case of cells in rapidly dividing tissues and cells in tissue-culture. In many organs, such as the liver, the cells divide very infrequently, and in this case there is evidence from studies of chromosome abnormalities that some forms of sub-lethal damage can persist for a large fraction of the animal's life span.

The cells of the liver can be induced to divide by partial hepatectomy or by injection of carbon tetrachloride. If this is done at varying intervals after irradiation, damaged cells can be identified by chromosome abnormalities. Curtis, Tilley and Cowley (Radiat. Res., 22, 730; 1964) found that the number of damaged chromosomes declined steadily with time after irradiation, for periods up to a year, and deduced that this was caused by intracellular repair as well as by elimination by cell division. 